\title{
Does a Blended Infant Massage-Parenting Enhancement Program Improve Infant Health Outcomes?
}

\author{
Luz S. Porter ${ }^{1 *}$ and Brian 0. Porter ${ }^{2}$ \\ ${ }^{1}$ College of Nursing, Florida International University, Miami, Florida 33199, USA \\ ${ }^{2}$ External Co-Investigator, Florida International University, Miami, Florida 33199, USA
}

Received: February 25, 2016; Accepted: April 13, 2016; Published: April 19, 2016

*Corresponding author: Luz S. Porter, College of Nursing, Florida International University, Miami, Florida 33199, USA, Tel: +1-954-367-3349; Fax No: +1-954-367-3349; E-mail: luzporter@comcast.net

\begin{abstract}
Purpose: This study aimed to determine whether a blended Infant Massage-Parenting Enhancement Program (IMPEP) improved infant health outcomes (growth, development, safety in home environment/infant safety, and immunizations) among infants of Substance-Addicted Mothers (SAMs) actively engaged in outpatient rehabilitation.

Methods: Designed as a randomized, three-group controlled trial testing two levels of psycho-educational intervention (IMPEP versus Parenting Enhancement Program [PEP]) and a control group (standard of care parenting resources), the study was conducted in two substance abuse centers in southeast Florida on a convenience sample of 138 infants. IMPEP or PEP classes were held weekly on Weeks 2-5, with data collected at Baseline, Week 6, and Week 12 via an Infant Detecto Beam Scale with standardized measuring stick, the Bayley Scales of Infant Development, and two instruments developed for this study--the Home Environment Safety Checklist and the Infant Immunization Status Checklist. Data analyses included descriptive and nonparametric inferential tests, using Kruskall-Wallis, ANOVA, post-hoc Wilcoxon Rank Sum, and Mann-Whitney U tests.
\end{abstract}

Results: Infants of IMPEP SAMs preferentially demonstrate greater growth than infants of PEP SAMs, as reflected in length, weight, and head circumference measures at 6 and 12 weeks postBaseline, while overall growth was observed chronologically in all study groups. There were no clinically meaningful differences among study groups in infant development, home and infant safety, as well as immunization status.

Conclusion: The findings suggest that IM blended into a parenting enhancement program has value-added effects in promoting infant growth but not in infant development, home and infant safety, and immunization status.

Keywords: Infant growth and development; Home environment safety; Infant safety; Immunization

\section{Introduction}

The adverse effects of perinatal substance abuse are complex, including the potential for physical and neurological impairment of the developing fetus and neonate. Failure to establish successful mother-infant interactions can result in impaired infant development and stunted growth [1-3]. We conducted a longitudinal, randomized, controlled study of recovering substance abusing mother-infant dyads for a 4-year period from 2006-2010. The purpose of the study was to evaluate the effects of a blended Infant-Massage Parenting Enhancement Program (IMPEP) on maternal and infant health outcomes. Grounded in Social Learning Theory [4], a detailed description of the fourweek IMPEP psycho-educational intervention has been published previously [5], and the effects of the program on maternal health outcomes have been reported, which included reduced parenting stress and depressive symptoms [6]. In addition to developing an effective and cost-efficient intervention for improving the health of recovering Substance- Abusing Mothers (SAMs) and their infants through the consistent nurturance and caretaking of these infants by their mothers, the goal of this study was to evaluate whether the IMPEP improves infant health outcomes, including growth (length, weight, head circumference), development (motor, mental, behavioral), home environmental safety and infant care safety at 6 , and 12 weeks post-Baseline, and immunization status at 12 weeks post-Baseline.

Babies of mothers who use cocaine and heroin are born addicted to these narcotics and typically suffer withdrawal symptoms after birth $[7,8]$. The most prominent attributes of such babies are low birth weight, smaller head circumference, and delayed physical, emotional, and neurological growth. They also tend to be withdrawn, non-responsive, irritable and difficult to console, with poor sleeping and feeding patterns $[9,10]$. Moreover, infants born to these mothers who discontinue substance use after the first trimester display abnormal neurobehavioral cluster scores on the Brazelton Neonatal Behavioral Assessment [11]. Thus, in utero exposure to drugs including alcohol, heroin, crack and powder cocaine inhalants, marihuana, and tobacco may result in a lifetime of physical and mental problems for the child [12].

SAMs may also display a reduced capacity for preventive care of their infants. Behavioral and home environmental characteristics have been reported to be predictive of the initiation of immunization $[13,14]$. Socio-demographic factors such as poverty are consistently linked to delayed completion of the primary vaccination series, as well as poor adherence to the 
immunization schedule for older children, particularly as family size increases [14,15]. As systematic immunization programs facilitate disease-prevention among populations at greatest risk of developing disease and consuming significant health care resources, parenting interventions targeting urban, low-income mothers that subsequently improve infant immunization rates would provide cost-effective benefits.

Moreover, programs such as the IMPEP which focus on ameliorating maternal-infant attachment through direct physical interaction and relationship-building between mother and child hold great promise for improving infant health outcomes. However, evaluation of such programs is lacking. We have previously reviewed [6] literature on the benefits to highrisk infants of Infant Massage (IM), as delivered by health care providers (primarily nurses). In this study, we hypothesized that infants of recovering SAMs who experience IM delivered by their mothers, who are specifically trained in IMPEP classes (behavioral-cognitive approach), will demonstrate better health outcomes (physical growth; motor, mental, and behavioral development), increased safety in the home environment and during infant care, and higher immunizations rates, as compared to the infants of mothers participating in PEP classes (cognitive approach) or exposed only to the standard level of parenting education typically provided in addiction treatment programs.

\section{Methodology}

\section{Study design}

The study was designed as a randomized, controlled threearm experimental trial with repeated measures over time of infant health outcomes to compare the efficacy of two levels of classroom-based psycho-educational parenting interventions, either with (IMPEP) or without IM (PEP), versus a standard of care educational control in infants who were evaluated onsite at their mothers' outpatient drug treatment facilities [6]. Clusters of 3 to 6 recovering SAM-infant dyads were randomly assigned within each study site to one of the three study groups (IMPEP, PEP, Control) in a 1:1:1 ratio. Infant body measurements, developmental status, and home environmental safety were assessed at Week 1 (Baseline).

This was followed by a 4-week intervention period with SAMinfant dyads of the IMPEP and PEP groups taking once weekly classes on a consistent (but separate) day and time at Weeks 2, 3, 4 and 5. Both IMPEP and PEP interventions were taught by the same two certified nurse practitioners, incorporating demonstration and supervised practice of IM techniques (IMPEP only), child care training (e.g., infant CPR), interactive group discussions, and question-and-answer sessions on parenting practices [6]. SAM-infant dyads of the control group had no specific group-based parenting or IM intervention, receiving only the standard of care parenting education materials offered to all recovering SAMs (including IMPEP and PEP participants) at their respective addiction treatment programs. At Week 6, a post intervention evaluation was completed with the same physiologic and developmental assessments, which were repeated at Week 12. A previous methodological article describes the strategies used to maximize data integrity and minimize between-group contamination in the study [16].

\section{Setting and sample}

The study was conducted from September 1, 2003 to August 31,2008 in two community addiction treatment centers in Miami-Dade and Broward Counties (Florida, USA), which serve a predominantly low-income client-base and offer residential addiction treatment services in an outpatient setting.

Inclusion criteria required mothers to have an infant 12 months of age or younger, speak and comprehend English, Spanish, or Creole, independently perform activities of daily living, maintain attention and follow directions, and willingly provide informed consent in writing. Only the mother's youngest child was enrolled in the study and comprised the motherinfant dyad. All SAMs registered at these sites were eligible for study participation, regardless of drugs of abuse (including alcohol), age, race/ethnicity, marital status, religion, education, or income level, although participation was entirely voluntary. A convenience sample of SAMs willing to undergo screening was selected by posting notices at each treatment center with information about the study and dates for on-site screening sessions, as well as through the encouragement by treatment center staff for voluntary participation.

A total of 170 recovering SAMs were screened for the study, with 32 mothers making no further contact with the study team (lost to follow-up) after the initial screening information session. As illustrated in Figure 1 [6], within-site randomization of consenting mothers was done in clusters of 3-6 mother-infant dyads (depending on the number of mothers who attended a given screening session) in a 1:1:1 ratio, yielding 62 IMPEP, 37 PEP, and 39 Control mother-infant pairs at Baseline. Of the initial 138 mother-infant dyads who consented and enrolled in the study, 17 dyads failed to complete the study by Week 12 , resulting in an overall retention rate of $87.7 \%$ (IMPEP $=82.3 \%$, PEP $=94.6 \%$, Control $=89.7 \%$ ) of infants with assessments at both Baseline and Week 12 . Assuming an attrition rate of $25.0 \%$, a Type I error of 0.05 , and a medium effect size $(0.25)$, a sample size of 52 per arm was originally predicted to provide $80.0 \%$ power to detect a within-subjects change of this magnitude following the IMPEP intervention [17]. However, the final sample of 121 infants at Week 12 did not meet this goal.

\section{Measurements and instruments}

An Infant Detecto Beam Weighing Scale with standardized measuring stick was used to measure the infant's weight in kilograms and length in centimeters. The Bayley Scales of Infant Development (BSID) $[18,19]$ was used to measure both physical and mental development. This is a standardized, norm-referenced instrument comprised of three sections: Mental Development Index; Psychomotor Development Index: and Behavior Rating Scale. The BSID has been increasingly used to evaluate infants and children from 1 to 42 months of age for both developmental delays and indicator of progress, with well characterized psychometric properties [19,20]. The Home Environment Safety Checklist, 
derived from the 2002 Easter Seals Home Safety Checklist, measured the level of infant safety in relation to both home environmental characteristics (Home Safety) and the quality of infant care (Safety in Infant Care). The Infant Immunization Status Checklist, based on the 2001 Florida Department of Health Immunization Guidelines and the 2001 recommendations of the Advisory Committee on Immunization Practices of the American Academy of Pediatrics and the American Academy of Family Physicians, systematically tracked the types and dates of vaccines received, allowing for the calculation of adherence rates to recommended infant immunization guidelines.

\section{Data Analysis}

Sample distribution of individual outcome variables was tested for normality using the Kolmogorov-Smirnov and Shapiro-Wilk tests, which indicated that none of the outcome variables met the assumption of normality. Therefore, nonparametric Kruskall-Wallis Analysis of Variance (ANOVA) with post-hoc Wilcoxon Rank Sum and Mann-Whitney U tests were used to compare ranked differences within and between treatment groups, respectively. All tests were conducted using the Statistical Package for Social Sciences Version 13.0, and results were considered significant at a two-sided significance level of $p<0.05$. Increases in Type I error due to multiplicity of testing were partly controlled by the use of Repeated Measures ANOVA to evaluate several pre-determined hypotheses comparing potential differences among the treatment groups on the following outcomes: increases in weight, length, and head circumference, individual developmental scores on the BSID, level of safety in the home environment and safety in infant care, and immunization adherence. Home environmental safety was quantified as the percent adherence to home safety guidelines, i.e., the total number of instituted home safety practices divided by the total number of Checklist Part One items. Safety in infant care was quantified as the percent adherence to instituted infant care practices divided by the number of total number of Checklist Part Two items. Adherence to standardized immunization guidelines was quantified as the percent adherence for each subject, i.e., the total number of immunizations received to date divided by the total number of individual recommended ageappropriate immunizations at each time point.

\section{Results}

The results reported in this manuscript are limited to infant health outcomes. An extensive analysis of the maternal health outcomes resulting from this study have been published previously [6].

\section{Demographics}

A detailed demographic description of the study sample has been reported previously [6] including both maternal and infant characteristics, based on the total number of mother-infant dyads enrolled in the study at Baseline (IMPEP $=62, \mathrm{PEP}=37$, Control = 39). In brief, infants ranged in age from 1 to 16 weeks (mean $\sim 4$ weeks) at the time of enrollment and were ethnically diverse (white $=40.6 \%$, black $=37.0 \%$, Hispanic $=16.7 \%$ ), with slightly more females (54.3\%). Most infants had been carried to fullterm $(89.7 \%)$, with the majority delivered via natural childbirth (59.5\%).

\section{Disposition}

As reported previously in detail following the 4-week intervention period [6], a minimal number of mother-infant dyads dropped out of the study, due to various factors that impacted the continued participation of the mothers, including relocation to another state, incarceration, and loss to followup. Overall drop-outs from Baseline to Week 12 were greater in number in the IMPEP (Baseline $=62$, Week $6=59$, Week $12=51$ ) group compared to the PEP (Baseline $=37$, Week $6=35$, Week $12=35$ ) and Control (Baseline $=39$, Week $6=35$, Week $12=35$ ) groups.

\section{Infant growth: length, weight, and head circumference}

Consistent with the hypothesis that the IMPEP intervention will preferentially achieve greater growth among IMPEP infants than PEP infants, we evaluated whether the IMPEP group at Weeks 6 and 12 demonstrated greater increases in length, weight and head circumference, compared to the PEP and Control groups. Summary statistics of infant growth parameters among study groups across assessment periods are depicted in Table 1, with results of the Kruskal-Wallis ANOVA for between and within group differences over time shown in Table 2.

As expected, overall growth was observed chronologically in all study groups. IMPEP infants showed significant growth in length (adjusted $\mathrm{z}=2.679, p=.007$ ), weight (adjusted $\mathrm{z}=$ 5.352, $p<.001$ ), and head circumference (adjusted $\mathrm{z}=7.307, p$ $<.001$ ) from Baseline to Week 6 and from Baseline to Week 12 (adjusted $\mathrm{z}=4.386, p<.001$; adjusted $\mathrm{z}=5.167, p<.001$; and adjusted $\mathrm{z}=7.039, p<.001$, respectively). Similarly, PEP infants showed significant growth in length and head circumference from Baseline to Week 6 (adjusted $\mathrm{z}=2.155, p=.031$; adjusted $\mathrm{z}=2.978, p=.003$, respectively) and in length, weight and head circumference from Baseline to Week 12 (adjusted $\mathrm{z}=3.098, p=$ .002 ; adjusted $\mathrm{z}=3.766, p<.001$; and adjusted $\mathrm{z}=4.465, p<.001$, respectively). The Control group also showed increases in weight and head circumference from Baseline to Week 6 and in length and head circumference from Baseline to Week 12. However, these gains in length, weight, and head circumference were generally less than those achieved by the IMPEP infants.

As an extension of the between-group Kruskal-Wallis ANOVA results shown in Table 2, post-hoc Mann-Whitney U tests indicated that IMPEP infants demonstrated significantly greater growth in weight (adjusted $\mathrm{z}=3.471, p=.001$ ) and head circumference (adjusted $\mathrm{z}=4.456, p<.001$ ) than PEP infants at the Week 6 post-intervention assessment. In addition, IMPEP infants showed significantly greater increases in head circumference (adjusted $\mathrm{z}=2.271, p=.023$ ) than PEP infants at Week 12. Therefore, the data lend support to the hypothesis that infants of IMPEP SAMs preferentially demonstrate greater growth than infants of PEP SAMs, as reflected in length, weight, and head circumference measures at 6 and 12 weeks post-Baseline, while as expected, 
Table 1: Descriptive Statistics by Study Group for Infant Growth Measures (Length, Weight, Head Circumference), Bayley Scales of Infant Development (Motor Development, Mental Development, Behavioral Rating), Home Environment Safety, and Safety in Infant Care-Mean (M) and Standard Deviation (SD).

\begin{tabular}{|c|c|c|c|c|c|c|c|c|c|}
\hline \multirow[t]{2}{*}{ Variable } & \multicolumn{3}{|c|}{ Baseline } & \multicolumn{3}{|c|}{ Week 6} & \multicolumn{3}{|c|}{ Week 12} \\
\hline & $\mathbf{N}$ & $\mathbf{M}$ & SD & $\mathbf{N}$ & M & SD & $\mathbf{N}$ & $\mathbf{M}$ & SD \\
\hline \multicolumn{10}{|c|}{ Length $(\mathrm{cm})$} \\
\hline IMPEP & 62 & 59.84 & 6.09 & 59 & 63.06 & 6.51 & 51 & 65.67 & 6.04 \\
\hline PEP & 37 & 60.57 & 7 & 35 & 63.91 & 6.91 & 35 & 65.53 & 6.01 \\
\hline Control & 39 & 60.51 & 6.54 & 35 & 62.1 & 6.02 & 35 & 64.76 & 6.16 \\
\hline \multicolumn{10}{|c|}{ Weight (lb) } \\
\hline IMPEP & 62 & 6.18 & 2.15 & 59 & 8.11 & 1.76 & 51 & 8.14 & 1.78 \\
\hline PEP & 35 & 6.37 & 2.29 & 33 & 6.52 & 2.32 & 35 & 8.47 & 1.66 \\
\hline Control & 39 & 6.02 & 1.55 & 35 & 7.92 & 1.73 & 35 & 7.91 & 1.44 \\
\hline \multicolumn{10}{|c|}{ Head Circumference (cm) } \\
\hline IMPEP & 62 & 40.38 & 2.83 & 59 & 44.56 & 1.99 & 51 & 44.66 & 2.1 \\
\hline PEP & 37 & 40.06 & 2.57 & 35 & 42.02 & 2.65 & 35 & 43.28 & 2.7 \\
\hline Control & 39 & 40.72 & 2.35 & 35 & 42.39 & 2.38 & 35 & 41.96 & 2.53 \\
\hline \multicolumn{10}{|c|}{ BSID Motor Development } \\
\hline IMPEP & 62 & 87.13 & 4.86 & 59 & 90.08 & 4.27 & 51 & 92.49 & 2.92 \\
\hline PEP & 37 & 87.27 & 4.45 & 35 & 89 & 3.82 & 35 & 92.03 & 3.85 \\
\hline Control & 39 & 87.64 & 4.46 & 35 & 90.17 & 3.76 & 35 & 90.57 & 3.58 \\
\hline \multicolumn{10}{|c|}{ BSID Mental Development } \\
\hline IMPEP & 62 & 88.31 & 3.54 & 59 & 92.22 & 2.7 & 51 & 90.73 & 2.78 \\
\hline PEP & 37 & 90.49 & 5.45 & 35 & 91.29 & 4.3 & 35 & 91.34 & 4.17 \\
\hline Control & 39 & 88.87 & 3.4 & 35 & 91.11 & 3.09 & 35 & 92 & 3.72 \\
\hline \multicolumn{10}{|c|}{ BSID Behavioral Rating } \\
\hline IMPEP & 62 & 88.45 & 3.15 & 59 & 92.42 & 3.94 & 51 & 90.39 & 2.71 \\
\hline PEP & 37 & 87.54 & 4.39 & 35 & 91.31 & 3.37 & 35 & 91.09 & 3.19 \\
\hline Control & 39 & 88.13 & 3.35 & 35 & 90.86 & 3.06 & 35 & 90.54 & 3.95 \\
\hline \multicolumn{10}{|c|}{ Home Environmental Safety } \\
\hline IMPEP & 62 & 84.39 & 13.7 & 58 & 85 & 16.79 & 51 & 85.63 & 17.08 \\
\hline PEP & 37 & 79.49 & 17.46 & 35 & 86.56 & 13.2 & 35 & 87.21 & 13.02 \\
\hline Control & 39 & 83.1 & 13.18 & 35 & 80.17 & 19.41 & 35 & 78.72 & 18.86 \\
\hline \multicolumn{10}{|c|}{ Safety in Infant Care } \\
\hline IMPEP & 62 & 9.74 & 1.62 & 54 & 10.3 & 1.38 & 51 & 10.86 & 0.94 \\
\hline PEP & 34 & 10.24 & 2.08 & 35 & 10 & 1.8 & 35 & 10.49 & 1.31 \\
\hline Control & 36 & 9.78 & 1.2 & 34 & 9.82 & 1.71 & 35 & 10.03 & 1.56 \\
\hline
\end{tabular}

overall growth was observed chronologically in all study groups.

\section{Infant development: Bayley scales of infant development}

Consistent with the hypothesis that IMPEP infants will display more advanced development than PEP infants, as reflected in overall motor, mental, and behavioral ratings on the Bayley Scales of Infant Development (BSID) at 6 and 12 weeks post-
Baseline, we evaluated whether the IMPEP group demonstrated greater gains compared to the PEP and Control groups at these time points. Summary statistics of BSID domain scores among study groups across assessment periods are depicted in Table 1, with the results of the Kruskal-Wallis ANOVA for between and within group differences over time shown in Table 2.

Within-group analyses showed significant improvements 
Table 2: Nonparametric Inferential Statistics by Study Group for Infant Growth Measures (Length, Weight, Head Circumference), Bayley Scales of Infant Development (Motor Development, Mental Development, Behavioral Rating), Home Environment Safety and Safety in Infant Care.

\begin{tabular}{|c|c|c|c|c|}
\hline \multicolumn{2}{|c|}{ Independent Variables } & \multirow[t]{2}{*}{ Kruskal-Wallis ANOVA } & \multirow[t]{2}{*}{ Degrees of freedom } & \multirow[t]{2}{*}{$p$} \\
\hline Length & & & & \\
\hline \multirow[t]{3}{*}{ Between Groups } & Baseline & 0.13 & 2,138 & 0.936 \\
\hline & Week 6 & 1.31 & 2,129 & 0.52 \\
\hline & Week 12 & 0.67 & 2,121 & 0.717 \\
\hline \multirow[t]{3}{*}{ Within Groups } & IMPEP & 20.5 & 2,173 & $<.001$ \\
\hline & PEP & 10.51 & 2,107 & 0.005 \\
\hline & Control & 8.65 & 2,109 & 0.013 \\
\hline \multicolumn{5}{|l|}{ Weight } \\
\hline \multirow[t]{3}{*}{ Between Groups } & Baseline & 0.09 & 2,136 & 0.955 \\
\hline & Week 6 & 12.93 & 2,127 & 0.002 \\
\hline & Week 12 & 2.88 & 2,121 & 0.237 \\
\hline \multirow[t]{3}{*}{ Within Groups } & IMPEP & 37.76 & 2,172 & $<.001$ \\
\hline & PEP & 17.13 & 2,103 & $<.001$ \\
\hline & Control & 27.81 & 2,109 & $<.001$ \\
\hline \multicolumn{5}{|c|}{ Head Circumference } \\
\hline \multirow[t]{3}{*}{ Between Groups } & Baseline & 1.42 & 2,138 & 0.492 \\
\hline & Week 6 & 26.85 & 2,129 & $<.001$ \\
\hline & Week 12 & 19.57 & 2,121 & $<.001$ \\
\hline \multirow[t]{3}{*}{ Within Groups } & IMPEP & 70.36 & 2,172 & $<.001$ \\
\hline & PEP & 21.67 & 2,107 & $<.001$ \\
\hline & Control & 8.85 & 2,109 & 0.012 \\
\hline \multicolumn{5}{|c|}{ BSID Motor Development } \\
\hline \multirow[t]{3}{*}{ Between Groups } & Baseline & 0.38 & 2,138 & 0.827 \\
\hline & Week 6 & 1.81 & 2,129 & 0.404 \\
\hline & Week 12 & 8.23 & 2,121 & 0.016 \\
\hline \multirow[t]{3}{*}{ Within Groups } & IMPEP & 38.51 & 2,172 & $<.001$ \\
\hline & PEP & 22.01 & 2,107 & $<.001$ \\
\hline & Control & 10.99 & 2,109 & 0.004 \\
\hline \multicolumn{5}{|c|}{ BSID Mental Development } \\
\hline \multirow[t]{3}{*}{ Between Groups } & Baseline & 4.71 & 2,138 & 0.095 \\
\hline & Week 6 & 2.86 & 2,129 & 0.24 \\
\hline & Week 12 & 4.87 & 2,121 & 0.088 \\
\hline \multirow[t]{3}{*}{ Within Groups } & IMPEP & 37.25 & 2,172 & $<.001$ \\
\hline & PEP & 0.94 & 2,107 & 0.626 \\
\hline & Control & 17.35 & 2,109 & $<.001$ \\
\hline \multicolumn{5}{|c|}{ BSID Behavioral Rating } \\
\hline \multirow[t]{3}{*}{ Between Groups } & Baseline & 1.21 & 2,138 & 0.545 \\
\hline & Week 6 & 9.29 & 2,129 & 0.01 \\
\hline & Week 12 & 1.35 & 2,121 & 0.509 \\
\hline \multirow[t]{3}{*}{ Within Groups } & IMPEP & 49.08 & 2,172 & $<.001$ \\
\hline & PEP & 18.24 & 2,107 & $<.001$ \\
\hline & Control & 13.27 & 2,109 & 0.001 \\
\hline
\end{tabular}




\begin{tabular}{|l|l|l|l|l|}
\hline Home Environment Safety & & & \\
\hline Between Groups & Baseline & 1.38 & 2,138 & 0.501 \\
\hline & Week 6 & 1.84 & 2,128 & 0.399 \\
\hline & Week 12 & 4.07 & 2,121 & 0.131 \\
\hline Within Groups & IMPEP & 1.37 & 2,171 & 0.503 \\
\hline & PEP & 4.68 & 2,107 & 0.096 \\
\hline & Control & 0.43 & 2,109 & 0.807 \\
\hline Safety in Infant Care & & & 0.034 \\
\hline Between Groups & Baseline & 6.76 & 2,132 & 0.027 \\
\hline & Week 6 & 7.19 & 2,126 & 0.81 \\
\hline & Week 12 & 0.42 & 2,120 & 0.046 \\
\hline Within Groups & IMPEP & 6.17 & 2,169 & 0.469 \\
\hline & PEP & 1.51 & 2,104 & 0.541 \\
\hline
\end{tabular}

for IMPEP infants in motor development (adjusted $\mathrm{z}=3.287, p$ $=.001$ ), mental development (adjusted $\mathrm{z}=5.875, p<.001$ ), and behavioral rating (adjusted $\mathrm{z}=6.631, p<.001$ ) from Baseline to Week 6 and in motor development (adjusted $\mathrm{z}=6.111, p<.001$ ), mental development (adjusted $\mathrm{z}=3.549, p<.001$ ), and behavioral rating (adjusted $\mathrm{z}=3.110, p=.002$ ) from Baseline to Week 12. PEP infants showed significant improvements in behavior rating from Baseline to Week 6 and both motor development and behavioral rating from Baseline to Week 12.

Between-group analyses demonstrated significant differences for motor development at Week 12 and behavioral rating at Week 6. In particular, post-hoc Mann-Whitney U tests showed that these significant between-group differences were largely due to IMPEP infants demonstrating greater motor development than Control infants at Week 12 and greater behavioral ratings at Week 6. However, the differences between IMPEP and PEP infants did not reach significance for any of the BSID variables at Week 6 or 12, with PEP infants noted to have significantly greater mental development than IMPEP infants at Baseline (adjusted $\mathrm{z}=2.140, p=.032$ ). Thus, based on these findings, the data did not support the hypothesis that the IMPEP intervention leads to greater advances in infant development compared to the PEP intervention alone, although IMPEP infants showed higher development scores than infants of mothers in the Control group exposed to standard of care parenting education.

\section{Home environmental safety and infant care safety}

To assess whether IMPEP infants will achieve greater child safety status than PEP infants, as reflected in greater home environmental safety scores on their mothers' self-report on the Home Environment Safety Checklist (i.e., home safety, infant care safety) at 6 and 12 weeks post-Baseline, we evaluated whether IMPEP infants had higher scores on this instrument. Summary descriptive statistics for this instrument among the study groups across assessment periods are depicted in Table 1, with results of the Kruskal-Wallis ANOVA for between and within group differences over time shown in Table 2.
Although there was some evidence that mothers in the IMPEP group increased their adherence to home environmental safety practices over time, significant differences were not observed among study groups in this domain of the Home Environment Safety Checklist. In contrast, significant differences were seen among groups in the level of safety in infant care among study groups at both Baseline and Week 6. In particular, post-hoc Mann-Whitney U tests demonstrated that IMPEP SAMs showed greater adherence to home environmental safety and safety in infant care than Control SAMs at Week 6 (adjusted $\mathrm{z}=2.698, p=$ .007), although this pattern was also seen at Baseline (adjusted $\mathrm{z}$ $=2.681, p=.007$ ) . Thus, the results did not support the hypothesis that the IMPEP intervention leads to greater child safety status than the PEP intervention alone, although improvements over standard parenting education (Control arm) were noted.

\section{Immunization status}

To assess whether IMPEP infants will display better immunization status than PEP infants, adherence to American Pediatric Association childhood immunization guidelines was evaluated among study groups at 6 and 12 weeks post-Baseline. Over the course of this 12-week study, all infants were up-todate in their immunization status, achieving $100 \%$ adherence to standard pediatric immunization guidelines across all groups. Thus, no group differences were observed, and the results did not support the hypothesis that IMPEP infants will preferentially demonstrate better immunization status than PEP infants.

\section{Discussion}

Child development theorists assert that the most important task of an infant's life is to establish a sense of basic trust and security in others, which typically becomes well established by one year of age and forms the foundation of trust and security for a child's future $[10,21,22]$. Establishing security in a primary caregiver is the most important task of an infant's life, affecting its actions, reactions, and future interpersonal relationship. The impact of low-cost, early intervention, such as the IMPEP, 
is high with respect to parent-child health promotion and holds great potential for expanding the knowledge base of family preservation and health enhancement among high-risk populations. Being held, caressed, and cuddled contribute to a loving and attentive environment during this critical period, which has a direct and measurable impact on infant growth and development [18,23-26]. Massage facilitates bonding between the parent and the infant, fostering the development of warm positive relationships [27-29]. Touching, hugging, holding, kissing, rocking, massaging, making eye contact, talking, and singing to infants are activities many parents regularly perform $[27,28]$; however, recovering SAMs may not be exposed to adequate parenting models that demonstrate such behaviors. A systematic parenting enhancement program for SAMs that incorporates infant massage techniques, such as the IMPEP, addresses this important parenting need, with potential benefits for both mother and child [25-28]. Nurse educators, nurses, nurse practitioners, public health professionals, and substance abuse service providers may use positive data generated from this study to design client tailored parenting programs, which blends IM as a both a health promotion and a therapeutic intervention.

The use of mothers as therapists to deliver IM was shown to be effective in two studies [30,31], and the findings of the current study further contribute to the database. Mothers typically learn different ways to stimulate their infants during home care to promote infant growth and development. The valueadded benefits of IM as integrated into a systematically planned parenting enhancement program were demonstrated on infant growth by the use of the IMPEP intervention, thus supporting the benefits of structured, psycho-educational parenting programs on the infants of recovering SAMs in outpatient residential treatment settings.

Of note, this study failed to support the hypotheses that IM would preferentially enhance infant development (motor, mental, or behavioral components), home environmental safety, safety in infant care, or infant immunization rates beyond a structured parenting enhancement program alone. This may have been because the duration of the study period with reportable results (12 weeks post-Baseline) was too short to detect differences in these dimensions of infant development or in long-term safety practices, such as infant immunization. In addition, the SAMinfant dyads enrolled in this study were housed during the entire study period in an addiction rehabilitation facility designed to accommodate both mothers and their children. As the intention of this study was not to alter the standard health maintenance practices of these treatment programs, such controlled residential setting may have contributed to a heightened attentiveness to environmental safety practices (e.g., electrical outlet covers, secure non-skid floor coverings, accessible fire extinguishers, emergency telephone numbers), as well as the close monitoring of immunization practices. In turn, these infant-related outcomes may have been influenced more by institutional policy and facility maintenance practices of the rehabilitation centers than by maternal attributes.

Despite the value-added benefits of the IMPEP being limited to physical growth outcomes in this study, these results in infants, coupled with our previously reported positive findings on maternal health outcomes [6], reinforce the understanding that developing and practicing skills in infant massage can facilitate recovering drug-addicted mothers to give and receive nurturing, with positive health impacts for both mother and child. This phenomenon has been observed in premature infants and infants born into withdrawal syndromes due to maternal substance abuse during pregnancy, when massaged by healthcare staff $[15,25,26]$. However, this study is the first to provide direct evidence of this same phenomenon being observed when drug-addicted mothers themselves are taught IM techniques by certified nurse practitioners through personalized training in small group settings with their own infants [6]. Although the absence of multivariate analysis in this study suggests correlational, rather than causal, associations, these findings nonetheless reinforce the assertion that the IMPEP intervention may provide both physical and emotional benefits to infants and the person providing the massage $[32,33]$. Thus, IM incorporated into basic parenting activities, such as proper infant feeding and sleeping practices and safety measures, holds strong promise for improved parenting effectiveness in high-risk populations.

\section{Conclusion}

The need for low-cost, effective parenting interventions for drug-addicted women that build upon the natural components of early mother-infant interactions are critical. Meeting the challenge of promoting healthy development of at-risk infants by enhancing the parenting effectiveness of their mothers, the positive impact of this study on infant growth rates builds a strong foundation for future development. Although as previously reported for this study [6], improvements in maternal attachment and mother-infant interaction could not be attributed to the IMPEP intervention; future research may contribute to a theory of change for the observed treatment benefits on infant growth, particularly with regard to the quality of the care giving relationship as a mediating variable. In addition, the IMPEP intervention should be tested across cultural boundaries, as has been done for the original PEP intervention [5,6,32], to establish the generalizability of these findings within the global community.

A major limitation of this study was the post-randomization attrition of mother-infant dyads, which is a recognized challenge in working with this difficult to access research population. In addition to limiting the final sample size and, therefore, statistical power, subject attrition restricted the observation period to 12 weeks, instead of the originally planned 48-week duration, given the imbalance in drop-out rates after Week 12 that precluded comparative analyses at later time points. Moreover, although the number of drop-outs between Baseline and Week 6 was limited (2-4 per group), drop-outs between Week 6 and Week 12 occurred only in the IMPEP group, which is a potential threat to the validity of our findings. Statistical comparisons at Baseline between SAM-infant pairs who dropped-out versus completed the study up to Week 12 were limited by the nonparametric distribution of outcome variables in this study. Thus, 
future longitudinal studies with larger sample sizes may address concerns with attrition, although such studies are operationally challenging in the high-risk SAM population.

\section{Acknowledgement}

We wish to thank all of the mothers and their infants who participated in the study, as well as the Susan B. Anthony Recovery Center and The Village in downtown Miami, which were the two drug rehabilitation centers that served as our study sites. We also wish to thank Claire Pryce, ARNP, and Marjorie Williams, ARNP, (data collectors), Vivian Bango-Sanchez, ARNP, and Bonnie Kissel, ARNP, (IMPEP/PEP instructors), Dr. Sachin Nunnewar (research assistant), Henriek Meier (administrative assistant and data processor), Paul Scibelli (data management consultant), and MINIMAX Consulting (statistical analysis). This study was supported by the Maternal and Child Health Bureau (Tile V, Social Security Act), Health Resources and Services Administration, US Department of Health and Human Services, Grant R40 MC 01063.

\section{Declaration}

\section{Conflict of interest}

The authors declare no conflicts of interest that may bias the study findings.

\section{Ethics approval}

Approval of the research protocol by the Florida International University Institutional Review Board and the chief executive officers of the collaborating addiction treatment centers were secured before initiating subject recruitment. Although participants were encouraged to attend all intervention classes and complete all follow-up assessments, study participation was entirely voluntary, and mothers and their infants were free to leave the study at any time, incurring no penalty or limitation in services from their respective treatment centers.

\section{References}

1. Van der Horst FCP. John Bowlby- From Psychoanalysis to Ethology Unraveling the roots of attachment Theory. United Kingdom: John Wiley \& Sons Lt. 2011.

2. Na Zhua Hua, Xie Hui Yun, Huang Jian Hua. The effect of infant massage on growth. New Journal of Traditional Chinese Medicine. 2005;37:6970 .

3. Ferber SG, Feldman R, Kohelet D, Kuint J, Dollberg S, Arbel E, et al. Massage therapy facilitates mother-infant interaction in premature infants. Infant Behavior and Development. 2005;28(1):74-81.

4. Bandura A. Toward a Psychology of Human Agency. Perspectives on Psychological Science. 2006;1(2):164-180. doi:10.1111/j.17456916.2006.00011.x.

5. Porter LS, Porter BO. A blended infant massage--parenting enhancement program for recovering substance-abusing mothers. Pediatr Nurs. 2004;30(5):363-72, 401

6. Porter LS, Porter BO, McCoy V, Bango-Sanchez V, Kissel B, Williams $M$, et al. Blended Infant Massage-Parenting Enhancement Program on Recovering Substance-Abusing Mothers' Parenting Stress, SelfEsteem, Depression, Maternal Attachment, and Mother-Infant
Interaction. Asian Nurs Res (Korean Soc Nurs Sci). 2015;9(4):318-27. doi: 10.1016/j.anr.2015.09.002.

7. Wong S, Ordean A, Kahan M. Substance use in pregnancy. J Obstet Gynaecol Can. 2011;33(4):367-84.

8. Singer LT, Arendt R, Minnes S, Farkas K, Salvator A, Kirchner HL, et al. Cognitive and motor outcomes of cocaine-exposed infants. JAMA. 2002;287(15):1952-60.

9. Patrick SW, Schumacher RE, Benneyworth BD, Krans EE, McAllister JM, Davis MM. Neonatal abstinence syndrome and associated health care expenditures: United States, 2000-2009. JAMA. 2012;307(18):193440. doi: 10.1001/jama.2012.3951.

10. Bowlby J. A secure base: Parent-child attachment and healthy human development. New York: Basic Books; 1988.

11. Voigt RG, Brown FR, Fraley JK, Llorente AM, Rozelle J, Turcich M, et al. Concurrent and predictive validity of the cognitive adaptive test/clinical linguistic and auditory milestone scale (CAT/CLAMS) and the Mental Developmental Index of the Bayley Scales of Infant Development. Clin Pediatr (Phila). 2003;42(5):427-32.

12.Semrud-Clikeman M, Teeter Ellison PA. Child Neuropsychology: Assessment and Interventions for Neurodevelopmental Disorders. 2nd Edition. New York: Springer. 2007.

13. UNICEF, World Bank. State of the world's vaccine and immunization. 3rd edition. Geneva: World Health Organization. 2009.

14. Etana B, Deressa W. Factors associated with complete immunization coverage in children aged 12-23 months in Ambo Woreda, Central Ethiopia. BMC Public Health. 2012;12:566. doi: 10.1186/1471-245812-566.

15. Ang JY, Lua JL, Mathur A, Thomas R, Asmar BI, Savasan S, et al. A randomized placebo-controlled trial of massage therapy on the immune system of preterm infants. Pediatrics. 2012;130(6):e154958. doi: 10.1542/peds.2012-0196.

16. Porter B, Porter L, McCoy V, Lima M, Pryce C, Nunnewar S. Methodological challenges in intervention studies. Nurse Res. 2009;16(2):43-63.

17. Cumming G. Understanding the new statistics: Effect Sizes, confidence intervals, and meta-analysis. New York, USA: Routledge. 2012.

18. Brazelton TB, Nugent JK. Neonatal behavioral assessments scale. $4^{\text {th }}$ Edition. London: Mac Keith Press; 2011.

19. Albers CA, Grieve AJ. The Bayley scales of infant development. Journal of Psycho-educational Assessment. 2007.

20. Claudine Amiel-Tison, Julie Gosselin. Neurological Development from Birth to Six Years: Guide for Examination and Evaluation. Baltimore, MD: Johns Hopkins University Press. 2001.

21. Landers MS, Sullivan RM. The development and neurobiology of infant attachment and fear. Dev Neurosci. 2012;34(2-3):101-14.

22. Ainsworth MD. Attachments beyond infancy. Am Psychol. 1989;44(4):709-16.

23. Underdown A, Barlow J, Chung V, Stewart-Brown S. Massage intervention for promoting mental and physical health in infants aged under 6 months. Cochrane Database Syst Rev. 2006;(4):CD005038.

24.SAMSHA. Addressing the needs of women and girls: Developing core competencies for mental health and substance abuse service professionals. 2011.

25. Vickers A, Ohlsson A, Lacy JB, Horsley A. Massage for promoting 
growth and development of preterm and/or low birth-weight infants. Cochrane Database Syst Rev. 2004;(2):CD000390.

26. Onozawa K, Glover V, Adams D, Modi N, Kumar RC. Infant massage improves mother-infant interaction for mothers with postnatal depression. J Affect Disord. 2001;63(1-3):201-7.

27. Vimala McClure. Infant massage. A handbook for loving parents. New York: Bantam Books. 2000.

28. Liu CL. The effect of touching on healthy infants' weight. Journal of Huaihai Medicine.2005;23:137.

29. Mullany LC, Darmstadt GL, Khatry SK, Tielsch JM. Traditional massage of newborns in Nepal: Implications for trials for improved practice. ] Trop Pediatr. 2005;51(2):82-6.

30. Ferber SG, Kuint J, Weller A, Feldman R, Dollberg S, Arbel E, et al
Massage therapy by mothers and trained professionals enhances weight gain in preterm infants. Early Hum Dev. 2002;67(1-2):37-45.

31. Singla DR, Kumbakumba E, Aboud FE. Effects of a parenting intervention to address both maternal psychological wellbeing and child development and growth in rural Uganda: A community-based, cluster randomised trial. Lancet Glob Health. 2015;3(8):e458-69. doi: 10.1016/S2214-109X(15)00099-6.

32. Porter LS, Youssef M, Shaaban I, Ibrahim W. Parenting enhancement among Egyptian mothers in a tertiary care setting. Pediatr Nurs. 1992;18(4):329-36.

33. Yousafzai AK, Rasheed MA, Rizvi A, Armstrong R, Bhutta ZA. Parenting Skills and Emotional Availability: A RCT. Pediatrics. 2015;135(5):e1247-57. doi: 10.1542/peds.2014-2335. 